\title{
Insulin Resistance in Polycystic Ovary Syndrome: Progress and Paradoxes
}

\author{
Aradhana M. Venkatesan, Andrea Dunaif, and Anne Corbould \\ The Division of Women's Health, The Departments of Medicine and of Obstetrics and Gynecology, \\ Brigham and Women's Hospital, 75 Francis Street, Boston, Massachusetts 02115
}

\begin{abstract}
Over the past 20 years, it has been clearly documented that 1 ) polycystic ovary syndrome (PCOS) has major metabolic sequelae related to insulin resistance and 2) insulin resistance plays an important role in the pathogenesis of the reproductive abnormalities of the disorder. Women with PCOS are at significantly increased risk of developing type 2 diabetes mellitus (DM). Studies in isolated adipocytes and in cultured skin fibroblasts from PCOS women have demonstrated intrinsic postbinding defects in insulin-mediated glucose metabolism. In fibroblasts, the mitogenic pathway of insulin action is intact, consistent with a selective defect in insulin signaling. While PCOS skeletal muscle is resistant to insulin in vivo, cultured muscle cells have normal insulin sensitivity, consistent with a major role of extrinsic factors in producing insulin resistance in this tissue. Excessive serine phosphorylation of the insulin receptor or downstream signaling proteins may be involved in the pathogenesis of insulin resistance in PCOS. The putative serine kinase is extrinsic to the insulin receptor but its identity is unknown. The explanations for tissue-specific and signaling pathway-specific differences in insulin action in PCOS are unknown but may involve differential roles of insulin receptor substrate (IRS)-1 and IRS-2 in insulin signal transduction.
\end{abstract}

\section{PCOS - A Disorder of Insulin Action and a Risk Factor for Type 2 Diabetes Mellitus}

Polycystic ovary syndrome (PCOS) is among the most-common endocrinopathies of premenopausal women, with a prevalence estimated at approximately 5 percent of this population (Knochenhauer et al., 1998). PCOS is characterized by hyperandrogenism, anovulatory infertility, and profound insulin resistance. In addition to playing an important role in the pathogenesis of the reproductive abnormalities of PCOS, insulin resistance has major metabolic consequences. Women with PCOS have an approximately seven-fold increased risk of developing type 2 diabetes mellitus (DM), compared to that of unaffected women (Legro et al., 1999).

The association of hyperandrogenism and diabetes was first described by Achard and Thiers in 1921 as "la diabete des femmes a barbe" (diabetes of bearded women) (Achard and Thiers, 1921). In 1980, Burghen and colleagues 
reported that obese women with PCOS had significantly elevated basal and postglucose-load insulin levels, compared to weight-matched control women, suggesting that the PCOS women were resistant to insulin (Burghen et al., 1980). Subsequent studies determined that hyperinsulinemia was a characteristic feature of women with PCOS, independent of obesity (Dunaif et al., 1987). In addition, Dunaif and colleagues found that obese women with PCOS had significantly increased glucose levels in response to an oral glucose challenge, compared with age- and weight-matched ovulatory control women (Dunaif et al., 1987). A study of 254 women with PCOS found nearly 40 percent of them to be glucose intolerant, with 31 percent having impaired glucose tolerance and 7.5 percent having type 2 DM (Legro et al., 1999) (Figure 1). These prevalences were significantly higher than those in age-, weight-, and ethnicity-matched control women as well as those in US-based population studies of premenopausal women. Rates of impaired glucose tolerance and type 2 DM were similar in PCOS women of diverse ethnic backgrounds, suggesting that PCOS per se is a more-important risk factor for glucose intolerance in young women than either race or ethnicity (Legro et al., 1999; Ehrmann et al., 1999).

In women with PCOS, basal insulin secretion is increased and hepatic insulin clearance is reduced, resulting in hyperinsulinemia (O'Meara et al., 1993). Insulin-stimulated glucose utilization is decreased by 35 to 40 percent in women with PCOS, independent of obesity, a decrease similar in magnitude to that seen in type 2 DM (Dunaif, 1997). Obesity and PCOS have a synergistic negative impact

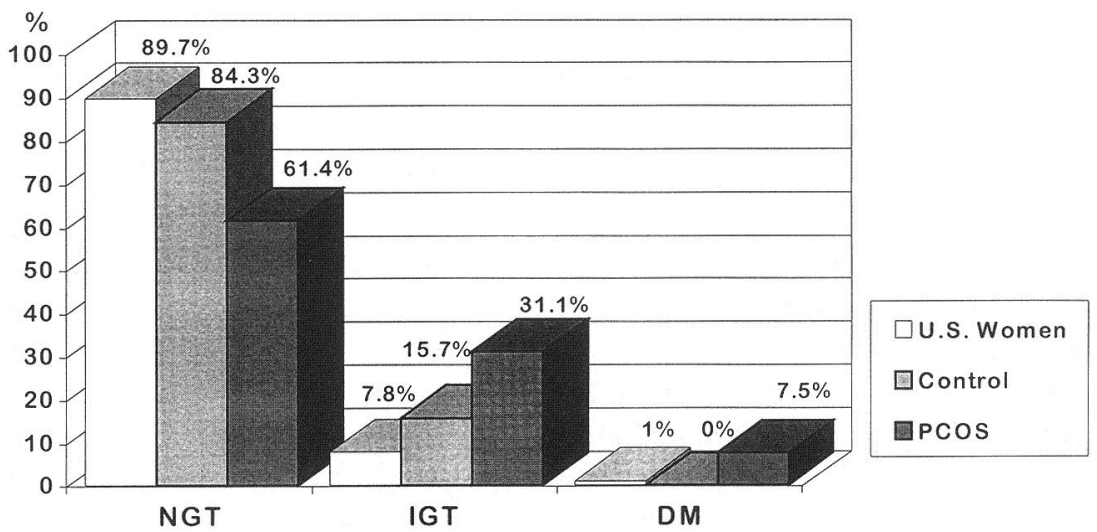

FIG. 1. Glucose tolerance in PCOS. Glucose tolerance was assessed by a 75-gram oral glucose tolerance test in women with PCOS; age-, weight-, and ethnicity-comparable reproductively normal control women; and US women, aged 20-44 years, from the Second National Health and Nutrition Survey (Legro et al., 1999). The prevalence rates for normal glucose tolerance (NGT), impaired glucose tolerance (IGT), and undiagnosed diabetes mellitus (DM) according to World Health Organization criteria are shown. [Reprinted with the permission of A. Dunaif.] 
on insulin sensitivity, so that hepatic insulin resistance is found only in PCOS women who are obese (Dunaif, 1997). Under normal circumstances, insulin secretion increases as insulin sensitivity decreases, in order to maintain glucose homeostasis. This relationship of insulin sensitivity and insulin secretion, known as the disposition index, is described by a hyperbolic curve (Bergman et al., 1996). In both nonobese and obese PCOS women, insulin secretion is inappropriately low for their degree of insulin resistance, suggesting the presence of pancreatic $\beta$-cell dysfunction in these patients (Ehrmann et al., 1995; Dunaif and Finegood, 1996) (Figure 2). Ehrmann and colleagues have demonstrated decreased postprandial insulin secretory responses as well as abnormalities in entrainment of insulin secretion to an oscillatory glucose infusion in PCOS women, consistent with $\beta$-cell dysfunction (Ehrmann et al., 1995). $\beta$-cell dysfunction can precede glucose intolerance in PCOS (Dunaif and Finegood, 1996).

Insulin resistance plays a role in the pathogenesis of the reproductive abnormalities characteristic of PCOS. Burghen and colleagues found a significant

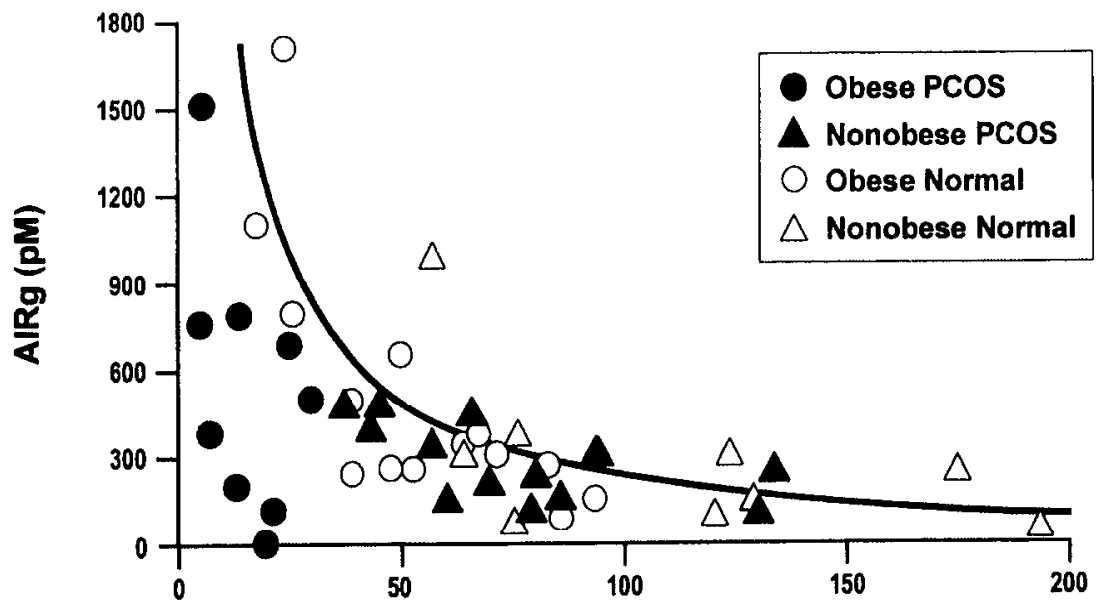

Insulin Sensitivity $\left(\mathrm{min}^{-1} /(\mathrm{nmol} / \mathrm{ml})\right)$

FIG. 2. The disposition index. Insulin sensitivity determined by minimal model analysis of a frequently sampled, intravenous glucose tolerance test is on the $x$-axis. Insulin secretion determined by the acute insulin response to an intravenous glucose load (AIRg) is on the $y$-axis. Under normal circumstances, the product of insulin sensitivity and insulin secretion, known as the disposition index, is constant and is described by a hyperbolic curve, as depicted. An early defect in the pathogenesis of diabetes is $\beta$-cell dysfunction, resulting in inappropriately low insulin secretion for the degree of insulin resistance. This is present in nonobese and obese women with PCOS, most of whom do not have DM, as can be seen in the figure where the disposition index is below the normal curve in PCOS women (solid symbols). [Reprinted with the permission of A. Dunaif.] 
positive correlation between insulin and androgen levels in their PCOS subjects, suggesting a possible causal relationship (Burghen et al., 1980). Insulin resistance is more severe in women with PCOS who are anovulatory than in women who are equally hyperandrogenemic but have normal cycles (Dunaif et al., 1987; Robinson et al., 1993), suggesting that insulin resistance contributes to anovulation. In obese women with PCOS who undergo weight loss, a reduction in basal and glucose-stimulated plasma insulin levels is associated with resumption of ovulatory cycles (Pasquali et al., 1989; Kiddy et al., 1992). Perhaps the most convincing of the lines of evidence linking insulin resistance and reproductive abnormalities have been studies utilizing insulin-sensitizing drugs to restore ovulation in women with PCOS (Dunaif et al., 1996; Nestler et al., 1998, 1999).

\section{Mechanisms of Insulin Resistance in PCOS}

\section{A. MOLECULAR EVENTS IN INSULIN SIGNALING}

Insulin is a hormone with diverse metabolic and mitogenic effects. In addition to promoting the synthesis and storage of carbohydrates, lipids, and proteins, insulin regulates cell growth and differentiation. The mechanisms controlling insulin receptor signal specificity are the subject of intense investigation (Virkamäki et al., 1999).

The insulin receptor belongs to a family of tyrosine kinase receptors, including the insulin-like growth factor-1 (IGF-1) receptor, with which it shares significant structural homology. (Other family members are epidermal growth factor, fibroblast growth factor, platelet-derived growth factor, and colony-stimulating factor-1 receptors) (De Fronzo, 1997). The insulin receptor is a heterotetramer consisting of two $\alpha, \beta$ dimers linked by disulfide bonds. The $\alpha$ subunit contains the ligand binding domain; the membrane-spanning $\beta$ subunit has an intracytoplasmic portion that possesses intrinsic tyrosine kinase activity (Cheatham and Kahn, 1995). Binding of insulin activates the receptor's kinase and leads to rapid autophosphorylation of specific tyrosine residues on the $\beta$ subunit (Saltiel, 1996). This results in tyrosine phosphorylation of insulin receptor substrate (IRS) molecules. The IRSs are a family of proteins, nine of which have been identified (Virkamäki et al., 1999; White and Yenush, 1998). These include IRS-1 through -4 , Gab-1, Shc (which has three isoforms), and p62 dok. All the proteins in this family bind transiently to the activated insulin receptor, during which time they are phosphorylated on several tyrosine sites. The tyrosine-phosphorylated sites on the insulin receptor substrates serve as binding sites for several SH2 domain proteins, including phosphatidylinositol 3-kinase (PI3-kinase). Activation of PI3kinase is a key step in the pathways ultimately resulting in stimulation of insulinmediated metabolic functions such as translocation of GLUT4 to the cell surface, 
leading to glucose uptake as well as glycogen synthesis. (Virkamäki et al., 1999; Saltiel, 1996; White and Yenush, 1998). PI3-kinase activation results in the phosphorylation of Akt, also known as protein kinase B (PKB), which, in turn, phosphorylates and inactivates glycogen synthase kinase-3 (GSK-3). GSK-3 tonically inhibits glycogen synthase activity. Thus, inhibition of GSK-3 activity results in glycogen synthase activation (Lawrence and Roach, 1997). Mitogenic actions of insulin (e.g., control of cell growth and differentiation) can occur independently of PI3-kinase activation via activation of the Ras-mitogen-activated protein (MAP) kinase pathway (Saltiel, 1996; White and Yenush, 1998) (Figure $3)$.

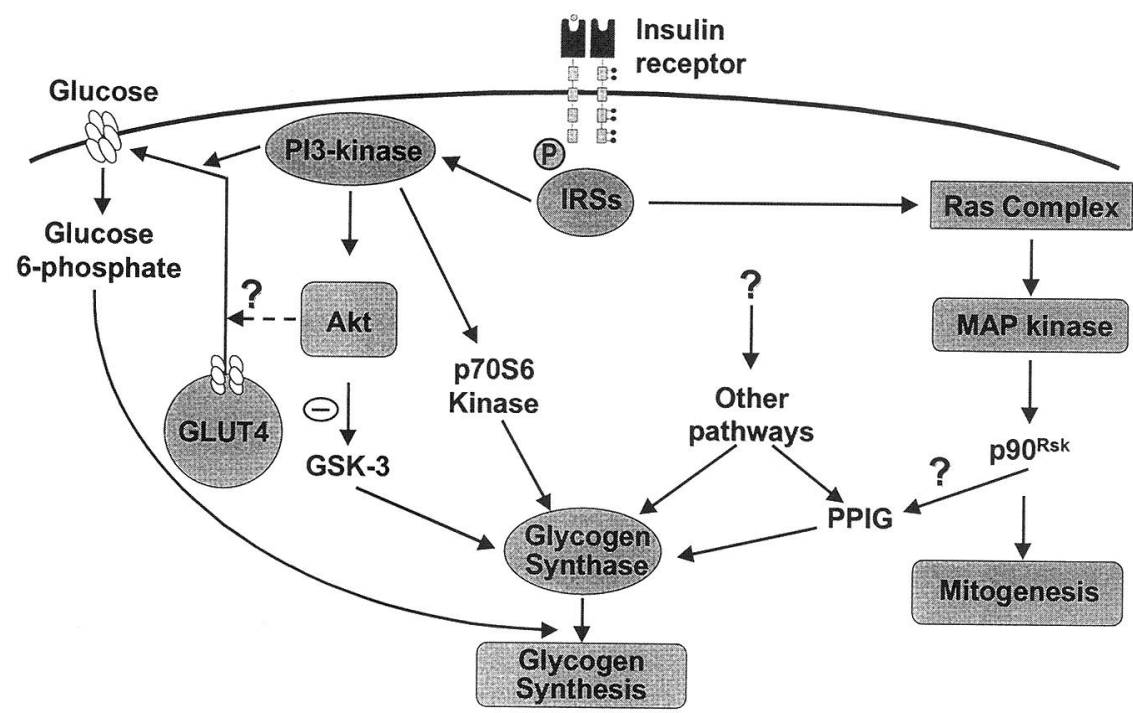

FIG. 3. Insulin receptor signaling. Insulin binding initiates signal transduction by inducing tyrosine autophosphorylation of the insulin receptor, which increases its protein kinase activity. The activated receptor tyrosine phosphorylates intracellular substrates such as insulin receptor substrates (IRSs) 1-4 and Shc (Src and collagen-homologous protein). Tyrosine phosphorylated sites on IRSs dock signaling and adaptor molecules propagating the signal. One important pathway is the activation of phosphatidylinositol 3-kinase (PI3-kinase), which ultimately leads to translocation of the insulinresponsive glucose transporter, GLUT4, and glucose uptake. PI3-kinase also results in the activation of Akt (also known as protein kinase B), which, in turn, phosphorylates and inactivates glycogen synthase kinase-3 (GSK-3). Inactivation of GSK-3 results in activation of glycogen synthase and glycogen synthesis. Other pathways (e.g., p70S6 kinase, glycogen-bound form of protein phosphatase 1 (PP1G)) may regulate glycogen synthesis. The insulin receptor can directly phosphorylate Shc, leading to the activation of the Ras signaling complex that results in MAP kinase activation and certain mitogenic actions of insulin. [Reprinted with the permission of A. Dunaif.] 


\section{B. INSULIN ACTION IN PCOS ADIPOCYTES}

Studies in adipocytes, a classic insulin target tissue, have demonstrated no abnormalities in insulin receptor number or affinity in PCOS women, when compared to appropriately weight-matched control women (Dunaif et al., 1992; Ciaraldi et al., 1992,1997). However, in the PCOS adipocytes, there was a striking and significant shift to the right in the dose-response curve for glucose uptake. Maximal rates of glucose transport were modestly but significantly decreased, compared to control women. This suggests the presence of a postbinding defect in insulin receptor-mediated signal transduction in PCOS. Decreased glucose transport in PCOS adipocytes has been shown to be secondary to a significant reduction in the abundance of GLUT4 glucose transporters (Rosenbaum et al., 1993). These defects in PCOS adipocytes occurred in the absence of glucose intolerance, obesity, or changes in waist-to-hip girth ratios. There was no significant relationship demonstrated with circulating levels of sex hormones (Dunaif et al., 1992). This suggests that the abnormalities of insulin action in PCOS adipocytes may reflect intrinsic rather than acquired defects.

\section{INSULIN ACTION IN PCOS FIBROBLASTS}

Although fibroblasts are not classic insulin target cells, defects in insulin receptor number and kinase activity in skin fibroblasts have reflected mutations in the insulin receptor in several of the rare syndromes of extreme insulin resistance (Taylor et al., 1992). Studies using cultured skin fibroblasts from women with PCOS have been performed to investigate the cellular mechanisms of insulin resistance in this disorder. Furthermore, by studying cells that have been removed from their in vivo environment for several generations, it is possible to assess insulin action without the confounding influences of the in vivo environment (e.g., hyperinsulinemia, hyperandrogenemia, hyperglycemia), all of which can produce insulin resistance (Dunaif, 1997). Presumably, signaling defects observed in cultured cells reflect intrinsic genetic defects in insulin action.

Consistent with experiments using isolated adipocytes, studies of insulin receptor binding in cultured skin fibroblasts from PCOS women have shown no differences in receptor number and affinity, compared with cultured cells from reproductively normal women (Dunaif et al., 1995). However, partially purified insulin receptors from cultured fibroblasts of nearly half of PCOS women (PCOSser) were noted to have markedly increased basal autophosphorylation with minimal further insulin-stimulated autophosphorylation. Phosphoamino acid analysis revealed that the increased basal phosphorylation represented primarily phosphoserine (Dunaif et al., 1995). Serine phosphorylation has been shown in several experimental systems to inhibit the intrinsic tyrosine kinase activity of the insulin receptor (White and Yenush, 1998). Consistent with this, the PCOS-ser insulin receptors demonstrated significantly reduced tyrosine kinase activity towards an 
artificial substrate, compared to partially purified insulin receptors from the fibroblasts of reproductively normal control women (Dunaif et al., 1995). This abnormal pattern of phosphorylation also has been detected in insulin receptors partially purified from PCOS skeletal muscle, a classic insulin target tissue, suggesting that this receptor abnormality may have relevance to the underlying pathogenesis of insulin resistance in PCOS (Dunaif et al., 1995).

No mutations have been detected in the coding region of the insulin receptor gene in women with PCOS (Sorbara et al., 1994; Dunaif et al., 1995; Talbot et al., 1996). Moreover, immunoprecipitation and mixing experiments have suggested that a factor extrinsic to the insulin receptor - presumably, a serine kinase - is responsible for the abnormal pattern of phosphorylation in PCOS fibroblasts (Dunaif et al, 1995). When control insulin receptors were mixed with wheat germ agglutinin eluates from the PCOS-ser fibroblast cell lines, a decrease in insulinstimulated tyrosine phosphorylation of the normal receptors was observed, along with an increase in serine phosphorylation of these receptors (Dunaif et al., 1995). These findings recently have been confirmed in studies of intact cells. $\mathrm{Li}$ and colleagues, using a highly sensitive ELISA technique to measure insulin receptor tyrosine phosphorylation in fibroblasts from PCOS and control women, showed a 33 percent decrease in insulin-stimulated receptor tyrosine phosphorylation in intact PCOS fibroblasts. However, no abnormality in insulin receptor tyrosine phosphorylation was observed when insulin receptors from solubilized PCOS fibroblasts were first immunocaptured and then stimulated with insulin. This finding is consistent with the existence of a factor extrinsic to the insulin receptor causing impaired signaling (Li et al., 2000).

Fibroblasts from approximately 50 percent of women with PCOS studied by Dunaif and colleagues (1995) manifested no abnormalities in insulin receptor phosphorylation, despite demonstrating in vivo insulin resistance comparable to PCOS-ser women. This observation suggests the existence of postreceptor abnormalities of insulin signaling in these women, perhaps at the level of IRS-1 phosphorylation or PI3-kinase activation. It is possible that the same factor responsible for excessive serine phosphorylation of the insulin receptor in the PCOS-ser women also phosphorylates downstream signaling proteins. Protein kinase $\mathrm{C}(\mathrm{PKC})$ is a candidate factor that can serine-phosphorylate the insulin receptor (White and Yenush, 1998) as well as IRS-l (De Fea and Roth, 1997).

The putative serine phosphorylation factor of PCOS is an example of insulin resistance secondary to extrinsic factors that interact with key proteins of the insulin-signaling cascade to inhibit signaling (Figure 4). Plasma cell differentiation factor-1 (PC-1) inhibits insulin receptor tyrosine kinase activity but is not associated with increased serine phosphorylation of the insulin receptor (Maddux et al., 1995). TNF- $\alpha$ appears to induce insulin resistance by serine phosphorylation of IRS-1, thus inhibiting propagation of the signal (Hotamisligil et al., 1996). 


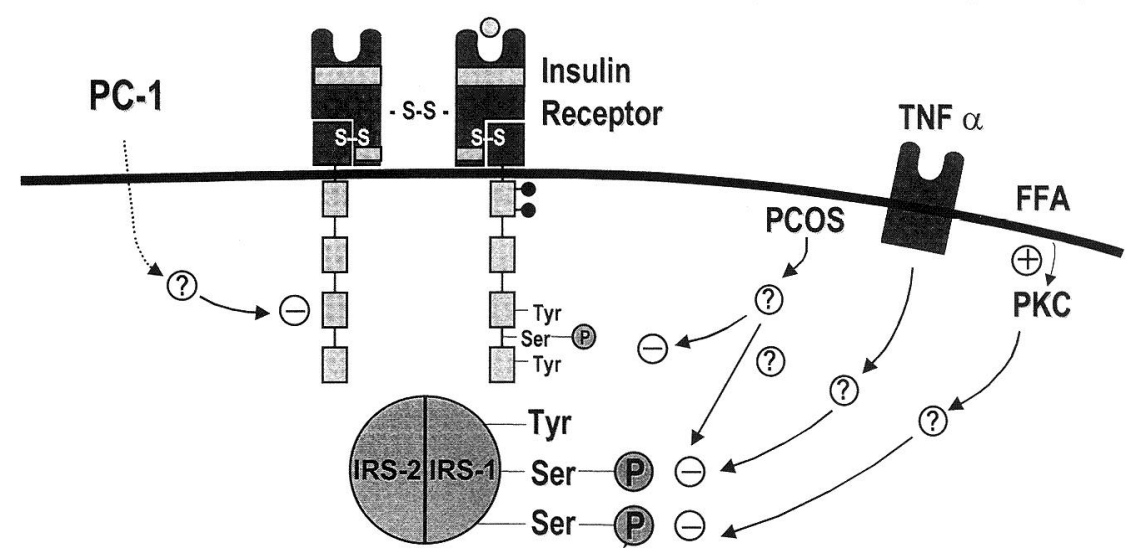

FIG. 4. Insulin resistance secondary to factors altering receptor signaling. Insulin resistance in cultured skin fibroblasts from approximately 50 percent of PCOS women appears to be secondary to a cell membrane-associated factor, presumably a serine/theronine kinase, that serine-phosphorylates the insulin receptor inhibiting signaling. Serine phosphorylation of IRS-1 appears to be the mechanism for TNF- $\alpha$-mediated insulin resistance. Free fatty acids can activate protein kinase C (PKC). PKCs can serine-phosphorylate IRS-1 and inhibit signaling. The membrane glycoprotein plasma cell differentiation factor also inhibits insulin receptor kinase activity but does not cause serine phosphorylation of the receptor. [Reprinted with the permission of A. Dunaif.]

Free fatty acids (FFAs) inhibit IRS-1 tyrosine phosphorylation and IRS-1-associated PI3-kinase activity, perhaps as a consequence of PKC-induced serine phosphorylation of IRS-1 (Griffin et al., 1999). There is evidence to suggest that hyperglycemia-induced insulin resistance is mediated by $\mathrm{PKC}$-induced serine phosphorylation of the insulin receptor (Pillay et al., 1996).

In order to determine whether the abnormalities observed in insulin receptor phosphorylation in PCOS fibroblasts were physiologically relevant, studies of insulin action in intact cells were performed. Insulin-stimulated glycogen synthesis was found to be significantly decreased in PCOS fibroblasts, whereas thymidine incorporation was unchanged, compared with control fibroblasts (Book and Dunaif, 1999). Thus, insulin resistance in these cells appears to be selective, affecting metabolic, but not mitogenic, pathways of insulin signaling. Similar findings have been reported in the cultured fibroblasts of patients with type 2 DM (Wells et al., 1993). IRS-1- and IRS-2-associated PI3-kinase activity was similar in cultured fibroblasts from PCOS and control women (Book and Dunaif, 1999; Venkatesan et al., 2000). Activation of Akt was similar in PCOS and control fibroblasts but GSK-3 phosphorylation was decreased in PCOS fibroblasts, consistent with an increase in GSK-3 activity (although this was not measured directly). Given that GSK-3 inhibits glycogen synthase, increased activity of GSK-3 
may account for the decreased glycogen synthesis observed in PCOS fibroblasts (Venkatesan et al., 2000). The relationship between abnormalities in phosphorylation of the insulin receptor and the downstream effects observed in PCOS fibroblasts is not clear.

\section{INSULIN ACTION IN PCOS SKELETAL MUSCLE}

Skeletal muscle is responsible for about 85 percent of insulin-stimulated glucose uptake in vivo (De Fronzo, 1997). Significant defects in insulin action have been demonstrated in the skeletal muscle of PCOS women, independent of obesity and hyperglycemia. In serial skeletal muscle biopsies obtained at baseline and during insulin infusion using the euglycemic clamp technique, insulin-stimulated activation of IRS-1-associated PI3-kinase was significantly decreased in PCOS skeletal muscle (Dunaif et al., in press). This occurred in parallel with decreased insulin-stimulated glucose uptake in vivo. The abundance of IRS- 1 and the p85 regulatory subunit of PI3-kinase was comparable in skeletal muscle from PCOS and control women. Thus, decreased IRS-1-associated PI3-kinase activation in PCOS muscle could not be accounted for by changes in the abundance of signaling proteins. Normal abundance of key insulin-signaling proteins also has been demonstrated in type $2 \mathrm{DM}$ without massive obesity (Bjornholm et al., 1997; Krook et al., 2000). However, alterations in abundance of insulin-signaling proteins have been described in other insulin-resistant states. In morbid obesity, the abundance of IRS-1 and p85 is decreased (Goodyear et al., 1995). In gestational $\mathrm{DM}$, the abundance of skeletal muscle insulin receptor is unchanged, whereas IRS- 1 abundance is decreased and IRS-2 and p85 are increased (Friedman et al., 1999).

These findings in PCOS skeletal muscle in vivo are consistent with inhibition of the insulin receptor tyrosine kinase by increased serine phosphorylation of the receptor that has previously been identified in PCOS cell-free systems (Dunaif $e t$ al., 1995). This mechanism may not be unique to PCOS. Recent studies suggest that increased insulin receptor serine/threonine phosphorylation in skeletal muscle may play a role in the pathogenesis of gestational DM (Shao et al., 2000). However, increased insulin receptor serine phosphorylation in the skeletal muscle of type 2 DM patients has not been demonstrated (Kellerer et al., 1995). Additionally, induction of serine phosphorylation of the insulin receptor in type $2 \mathrm{DM}$ may be secondary to hyperglycemia (Pillay et al., 1996), rather than an intrinsic defect.

All of these studies of PCOS and other insulin-resistant states used muscle biopsies or freshly isolated muscle strips. It is unclear whether the defects in insulin signaling that have been demonstrated in these studies are intrinsic to muscle tissue (i.e., genetically determined) or are acquired secondary to in vivo environmental factors such as hyperinsulinemia, increased circulating FFAs, or, 
in diabetic individuals, hyperglycemia. Another possibility is that both genetic and environmental influences on insulin signaling in skeletal muscle are important in the pathogenesis of insulin resistance, with underlying genetic defects in muscle conferring increased susceptibility to environmental factors.

Human skeletal muscle cells grown in culture show many of the characteristics of mature skeletal muscle in vivo. Therefore, this represents an excellent system for the study of muscle cells that have been removed from the in vivo environment (Sarabia et al., 1992; Henry et al., 1995). In contrast to the findings in PCOS skeletal muscle in vivo, our preliminary studies of culturcd muscle cclls of obese, nondiabetic PCOS women and weight-matched controls showed normal insulin responsiveness of glucose transport and glycogen synthesis in the PCOS muscle (unpublished data). These observations are consistent with a major role of environmental factors in the pathogenesis of insulin resistance in PCOS. Likewise, a recent study comparing insulin action and signaling in cultured skeletal muscle cells from normoglycemic, lean, insulin-resistant, and insulin-sensitive first-degree relatives of type $2 \mathrm{DM}$ patients failed to detect significant differences (Krützfeldt et al., 2000). However, a similar study using cultured skeletal muscle cells showed defects in insulin-stimulated glucose uplake in approximately half of overweight insulin-resistant, nondiabetic, first-degree relatives of type 2 diabetic patients, compared with lean control subjects (Jackson et al., 2000). Abnormalities in insulin action have been demonstrated in cultured skeletal muscle cells from patients with type 2 DM (Henry et al., 1995, 1996). However, it is not clear whether these defects are intrinsic or acquired, since it is possible that chronic hyperinsulinemia and/or hyperglycemia in vivo may induce nonreversible changes in insulin action. In insulin-resistant, nondiabetic Pima Indians, insulin action in cultured skeletal muscle cells was positively correlated with in vivo measures of insulin action, suggesting that genetic background may be relevant (Thompson et al., 1996; Mott et al., 1998; Youngren et al., 1999).

The candidate circulating factor that could produce skeletal muscle insulin resistance that has received the most attention is FFA. Circulating levels of FFAs correlate with in vivo measures of insulin resistance in first-degree relatives of individuals with type 2 DM (Gulli et al., 1992). Recent studies have shown that the administration of FFA to healthy subjects can produce insulin resistance and defects in muscle IRS-1-associated PI3-kinase activation (Dresner et al., 1999). Rodent studies suggest that this may be mediated via PKC-theta activation (Griffin et al., 1999). FFAs also appear to modulate hepatic glucose output (Bergman and Mittelman, 1998). These dual effects of FFA - to increase hepatic glucose production and to decrease muscle insulin sensitivity - have led Bergman to propose the "single-gateway hypothesis" (Bergman and Mittelman, 1998). According to this hypothesis, increased adipocyte lipolysis accounts for hepatic and peripheral insulin resistance. Adipocyte lipolysis could be increased by resistance to the antilipolytic actions of insulin and/or an increase in lipolytic stimuli (i.e., 
catecholamines), sensitivity to these stimuli, and/or the amount of lipolytically active visceral fat. FFA levels are increased in obese PCOS women and have not been studied in insulin-resistant, nonobese PCOS women (Holte et al., 1994; Robinson et al., 1996). Another circulating factor that could produce peripheral insulin resistance is TNF- $\alpha$ (Hotamisligil, 1999). One study has reported TNF- $\alpha$ clevations in PCOS (Naz et al., 1995).

\section{Insulin Resistance in PCOS: Progress and Paradoxes}

Insulin resistance in PCOS is associated with a postbinding defect in insulin receptor signaling that has been demonstrated in isolated adipocytes, cultured fibroblasts, and skeletal muscle in vivo. Current evidence suggests that constitutively increased serine phosphorylation of the insulin receptor or downstream key signaling proteins are involved in the pathogenesis of insulin resistance in PCOS. The factor responsible for this serine phosphorylation remains to be identified. Recent data suggest that this mechanism of induction of insulin resistance may not be unique to PCOS.

How this increase in serine phosphorylation of the insulin receptor or downstream proteins leads to a selective defect affecting only the metabolic pathway of insulin action in cultured PCOS skin fibroblasts is unclear. The mechanism may involve differential roles of IRS- 1 and IRS-2 in mediating the metabolic and mitogenic effects of insulin, in addition to abnormal interaction of the IRSs with the insulin receptor. The latter recently has been demonstrated in muscle cells expressing an insulin receptor containing a point mutation in the $\beta$ subunit (Arg1152Gln). This mutant receptor constitutively activates the metabolic pathway of insulin action, although mitogenic responses to insulin are normal (Petruzziello et al., 1993; Miele et al., 1999). In L6 muscle cells expressing the mutant receptor IRS-2, which was constitutively tyrosine phosphorylated and showed increased binding to the insulin receptor, mediated insulin regulation of glucose metabolism. In contrast, IRS-1 showed normal phosphorylation and mediated insulin mitogenic signals.

Tissue differences in insulin sensitivity may be present in PCOS, as reported by Ciaraldi and colleagues (1998). Isolated adipocytes and cultured skin fibroblasts from PCOS women demonstrate resistance to the metabolic effects of insulin. Conversely, preliminary studies of cultured PCOS skeletal muscle cells suggest that these cells are insulin sensitive, despite being insulin resistant in vivo. A possible explanation for these tissue-specific differences is that the abnormalities in insulin action in cultured fibroblasts and in isolated adipocytes reflect intrinsic defects. However, insulin resistance in skeletal muscle is either secondary to the in vivo environment or else interaction with environmental factors is required for an underlying genetic defect in insulin signaling to be manifested. The 
demonstration of abnormalities in insulin signaling in adipocytes of PCOS women is in keeping with the single-gateway hypothesis that proposes that increased adipocyte lipolysis, leading to increased circulating FFA levels, is the primary defect in insulin-resistant states. Tissue-specific differences in the roles of the IRS-1 and -2 in mediation of insulin signaling also may be relevant. Recent experiments using knockout mice have shown that IRS-1 plays a prominent role in insulin action in skeletal muscle and IRS-2 in liver (Higaki et al., 1999; Kido et al., 2000). Studies in human muscle appear to support these findings (Kim et al., 1999).

\section{Summary}

Women with PCOS are at substantially increased risk of developing impaired glucose tolerance and type $2 \mathrm{DM}$ at a young age. This risk is conferred by profound peripheral insulin resistance and defects in insulin secretion. The major abnormality in insulin action in PCOS is a postbinding defect in insulin signaling. This appears to be secondary to a factor extrinsic to the receptor that modulates its signaling and perhaps the signaling transduced by IRSs. Our preliminary studies suggest that not all tissues are intrinsically insulin resistant in PCOS. Skeletal muscle has acquired defects in insulin action. Hence, it is possible that insulin resistance in PCOS adipocytes leads to increased circulating FFA levels that, in turn, produce peripheral insulin resistance. Other circulating factors (e.g., TNF- $\alpha$ ) may contribute to insulin resistance in PCOS. Elucidating the pathogenesis of insulin resistance in PCOS will provide insight into an important cause of type $2 \mathrm{DM}$.

\section{REFERENCES}

Achard, C., and Thiers, J. (1921). Bull. Acad. Natl. Med. (Paris) 86, 51-64.

Bcrgman, R.N., and Mittclman, S.D. (1998). J. Basic. Clin. Physiol. Pharmacol. 9, $205-221$.

Bergman, R.N., Watanabe, R., Rebrin, K., Ader, M., and Steil., G. (1996). Diabet. Med. 13, S67-S77.

Björnholm, M., Kawano, Y., Lehtihet, M., and Zierath, J.R. (1997). Diabetes 46, 524-527.

Book, C.B., and Dunaif, A. (1999). J. Clin. Endocrinol. Metab. 84, 3110-3116.

Burghen, G.A., Givens, J.R, and Kitabchi, A.E. (1980). J. Clin. Endocrinol. Metab. 50, 113-116.

Cheatham, B., and Kahn, C.R. (1995). Endocr Rev. 16, 117-142.

Ciaraldi, T.P., El-Roeiy, A., Madar, Z., Reichart, D., Olefsky, J.M., and Yen, S.S.C. (1992). J. Clin. Endocrinol. Metab. 75, 577-583.

Ciaraldi, T.P., Morales, A.J., Hickman, M.G., Odom-Ford, R., Olefsky, J.M., and Yen, S.S.C. (1997). J. Clin. Endocrinol. Metab. 82, 1421-1425.

Ciaraldi, T.P., Morales, A.J., Hickman, M.G., Odom-Ford, R., Yen, S.S.C., and Olefsky, J.M. (1998). Metabolism 47, 940-946.

De Fea, K., and Roth, R.A. (1997). Biochemistry 36, 12939-12947.

De Fronzo, R.A. (1997). Diabetes Rev. 5, 177-269. 
Dresner, A., Laurant, D., Marcucci, M., Griffin, M.E., Dufour, S., Cline, G.W., Slezak, L.A., Andersen, D.K., Hundal, R.S., Rothman, D.L., Falk Petersen, K., and Shulman, G.I. (1999). J. Clin. Invest. $103,253-259$.

Dunaif, A. (1997). Endocr Rev. 18, 774-800.

Dunaif, A., and Finegood, D.T. (1996). J. Clin. Endocrinol. Metab. 81, 942-947.

Dunaif, A., Graf, M., Mandeli, J., Laumas, V., and Dobrjansky, A. (1987). J. Clin. Endocrinol. Metab. 65, 499-507.

Dunaif, A., Futterweit, W., Segal, K.R., Shelley, D.R., Green, G., Dobrjansky, A., and Licholai, T (1992). Diabetes 41, 1257-1266.

Dunaif, A., Xia, J., Book, C.B., Schenker, E. and Tang, Z. (1995). J. Clin. Invest. 96, 801-810.

Dunaif, A., Scott, D., Finegood, D., Quintana, B., and Whitcomb, R. (1996). J. Clin. Endocrinol. Metab. 81, 3299-3306.

Dunaif, A., Wu, X., Lee, A., and Diamanti-Kandarakis, E. (2001). Am J. Physiol., in press.

Fhrmann, D.A., Sturis, I, Ryrne, M.M., Karrison, T., Rosenfield, R.L., and Polonsky, K.S. (1995). J. Clin. Invest. 69, 520-527.

Ehrmann, D.A., Barnes, R.B., Rosenfield, R.L., Cavaghan, M.K., and Imperial, J. (1999). Diabetes Care $22,141-146$.

Friedman, J.E., Ishizuka, T., Shao, J., Huston, L., Highman, T., and Catalano, P. (1999). Diabetes 48, 1807-1814.

Goodyear, L.J., Giorgino, F., Sherman, L.A., Carey, J., Smith, R.J., and Dohm, G.L. (1995). J. Clin. Invest. 95, 2195-2204.

Griffin, M.E., Marcucci, M.J., Cline, G.W., Bell, K., Barucci, N., Lee, D., Goodyear, L.J., Kraegen, E.W., White, M.F., and Shulman, G.I. (1999). Diabetes 48, 1270-1274.

Gulli, G., Ferrannini E., Stern, M., Haffner, S., and DeFronzo, R.A. (1992). Diabetes 41, 1575-1586.

Henry, R.R., Abrams, L., Nikoulina, S., and Ciaraldi, T.P. (1995). Diabetes 44, 939-946.

Henry, R.R., Ciaraldi, T.P., Abrams-Carter, L., Mudaliar, S., Park, K.S., and Nikoulina, S.E. (1996). J. Clin. Invest. 98, 1231-1236.

Higaki, Y., Wojtaszewski, J.F., Hirshman, M.F., Withers, D.J., Towery, H., White, M.F., and Goodyear, L.J. (1999). J. Biol. Chem. 274, 20791-20795.

Holte, J., Bergh, T., Berne, C., and Lithell., H. (1994). Clin. Endocrinol. (Oxf.) 41, 463-471.

Hotamisligil, G.S. (1999). Exp. Clin. Endocrinol. Diabetes 107, 119-125.

Hotamisligil, G.S., Peraldi, P., Budavari, A., Ellis, R., White, M.F., and Spicgelman, B.M. (1996). Science 271, 665-668.

Jackson, S., Bagstaff, S.M., Lynn, S., Yeaman, S.J., Turnbull, D.M., and Walker, M. (2000). Diabetes 49, $1169-1177$.

Kellerer, M., Coghlan, M., Capp, E., Muhlhofer, A., Kroder, G., Mosthaf, L., Galante, P., Siddle, K., and Häring, H.U. (1995). J. Clin. Invest. 96, 6-11.

Kiddy, D.S., Hamilton-Fairley, D., Bush, A., Short, F., Anyaoku, V., Reed, M.J., and Franks, S. (1992). Clin. Endocrinol. 36, 105-111.

Kido, Y., Burks, D.J., Withers, D., Bruning, J.C., Kahn, C.R., White, M.F., and Accili, D. (2000). $J$. Clin. Invest. 105, 199-205.

Kim, Y.-B., Nikoulina, S.E., Ciaraldi, T.P., Henry, R.R., and Kahn, B.B. (1999). J. Clin. Invest. 104, 733-741.

Knochenhauer, E.S., Key, J., Kahsar-Miller, M., Waggoner, W., Boots, L.R., and Azziz, R. (1998). J. Clin. Endocrinol. Metab. 83, 3078-3082.

Krook, A., Bjornholm, M., Galuska, D., Jiang, X.J., Fahlman, R., Myers, M.G. Jr., Wallberg-Henriksson, H., and Zierath, J.R. (2000). Diabetes 49, 284-292.

Krützfeldt, J., Kausch, C., Volk, A., Klein, H.H., Rett, K., Häring, H.-U., and Stumvoll, M. (2000). Diabetes 49, 992-998.

Lawrence, J.C.J., and Roach, P.J. (1997). Diabetes 46, $541-547$. 
Legro, R.S., Kunselman, A., Dodson, W.C., and Dunaif, A. (1999). J. Clin. Endocrinol. Metab. 84, $165 \cdot 169$.

Li, M., Youngren, J.F., Maddux, B.A., Dunaif, A., Thomas, A., Zhang, B., and Goldfine, I.D. (2000). In "Program \& Abstracts of the 82nd Annual Meeting of The Endocrine Society," June 2000, Toronto, Canada, abstract 2331, p. 563.

Maddux, B.A., Sbraccia, P., Kumakura, S., Sasson, S., Youngren, J., Fisher, A., Spencer, S., Grupe, A., Henzel, W., Stewart, T.A., Reaven, G.M., and Goldfine, I.D. (1995). Nature 373, 448-451.

Miele, C., Caruso, M., Calleja, V., Auricchio, R., Oriente, F., Formisano, P., Condorelli, G., Cafieri, A., Sawka-Verhelle, D., Van Obberghen, E., and Beguinot, F. (1999). J. Biol. Chem. 274, 3094-3102.

Mott, D.M., Pratley, R.E., and Bogardus, C. (1998). J. Clin. Invest. 101, 2252-2256.

Naz, R.K., Thurston, D., and Santoro, N. (1995). Am. J. Reprod. Immunol. 34, 170-175.

Nestler, J.E., Jakubowicz, D.J., Evans, W.S., and Pasquali, R. (1998). N. Engl. J. Med. 338, 1876-1880.

Nestler, J.E., Jakubowicz, D.J., Reamer, P., Gunn, R.D., and Allan, G. (1999). N. Engl. J. Med. 340, 1314-1320.

O'Meara, N.M., Blackman, J.D., Ehrmann, D.A., Barnes, R.B., Jaspan, J.B., Rosenfield, R.L., and Polonsky, K.S. (1993). J. Clin. Endocrinol. Metab. 76, 1241-1247.

Pasquali, R., Antenucci, D., Casimirri, F., Venturoli, S., Paradisi, R., Fabbri, R., Balestra, V., Melchionda, N., and Barbara, L. (1989). J. Clin. Endocrinol. Metab. 68, 173-179.

Pillay, T.S., Xiao, S., and Olefsky, J.M. (1996). J. Clin. Invest. 97, 613-620.

Petruzziello, A., Formisano, P., Miele, C., Di Finizio, B., Riccardi, G., Ferrara, A., Beguinot, L., and Beguinot, F. (1993). J. Clin. Endocrinol. Metab. 77, 409-412.

Robinson, S., Kiddy, D., Gelding, S.V., Willis, D., Niththyananthan, R., Bush, A., Johnston, D.G., and Franks, S. (1993). Clin. Endocrinol. (Oxf.) 39, 351-355.

Robinson, S., Henderson, A.D., Gelding, S.V., Kiddy, D., Niththyananthan, R., Bush, A., Richmond, W., Johnston, D.G., and Franks, S. (1996). Clin. Endocrinol. (Oxf.) 44, 277-284.

Rosenbaum, D., Ilaber, R., and Dunaif, A. (1993). Am. J. Physiol. 264, E197-E202.

Saltiel, A.R. (1996). Am. J. Physiol. 270, E375-E385.

Sarabia, V., Lam, L., Burdett, E., Leiter, L.A., and Klip, A. (1992). J. Clin. Invest. 90, 1386-1395.

Shao, J., Catalano, P.M., Yamashita, H., Ruyter, I., Smith, S., Youngren, J., and Friedman, J.E. (2000). Diabetes 49, 603-610.

Sorbara, L.R., Tang, Z., Cama, A., Xia, J., Schenker, E., Kohanski, R.A., Poretsky, L., Koller, E., Taylor, S.I., and Dunaif, A. (1994). Metabolism 43, 1568-1574.

Talbot, J.A., Bicknell, E.J., Rajkhowa, M., Krook, A., O'Rahilly, S., and Clayton, R.N. (1996). J. Clin. Endocrinol. Metab. 81, 1979-1983.

Taylor, S.I., Cama, A., Accili, D., Barbetti, F., Quon, M.J., de la Luz Sierra, M., Suzuki, Y., Koller, E., Levy-Toledano, R., Wertheimer, E., Moncada, V.Y., Kadowaki, H., and Kadowaki, T. (1992). Endocr. Rev. 13, 566-595.

Thompson, D.B., Pratley, R., and Ossowski, V. (1996). J. Clin. Invest. 98, 2346-2350.

Venkatesan, A., Corbould, A., and Dunaif, A. (2000). In "Program \& Abstracts of the 82nd Annual Meeting of The Endocrine Society," June 2000, Toronto, Canada, abstract 330, p 88.

Virkamaki, A., Ueki, K., and Kahn, C.R. (1999). J. Clin. Invest. 103, 931-943.

Wells, A.M., Sutcliffe, I.C., Johnson, A.B., and Taylor, R. (1993). Diabetes 42, 583-589.

White, M.F., and Yenush, L. (1998). Curr. Top. Microbiol. Immunol. 228, 179-208.

Youngren, J.F., Goldfine, I.D., and Pratley, R.E. (1999). Am. J. Physiol. 276, E990-E994. 